\title{
Ventriculoperitoneal Shunt-Associated Vancomycin-Resistant Meningitis Complicating a Patient Undergoing Plasmapheresis for Presumed Systemic Lupus Erythematosus-Associated Transverse Myelitis
}

\author{
Adam John Covach ${ }^{1}$ William Nicholas Rose ${ }^{1}$ \\ ${ }^{1}$ Department of Pathology, University of Wisconsin Hospital and \\ Clinics, Madison, Wisconsin

\begin{abstract}
Address for correspondence William Nicholas Rose, MD, Department of Pathology, University of Wisconsin Hospital and Clinics, 600 Highland Ave, Madison, WI 53792 (e-mail: acovach@uwhealth.org).
\end{abstract}

J Neurol Surg Rep 2017;78:e123-e124.

\author{
Abstract \\ Keywords \\ - vancomycin-resistant \\ meningitis \\ - ventriculoperitoneal \\ shunt \\ - nosocomial infection \\ - transverse myelitis \\ - systemic lupus \\ erythematosus \\ - plasmapheresis
}

Objectives We report a patient who had a working diagnosis of transverse myelitis secondary to a lupus exacerbation. As his clinical course evolved, continued vigilance clarified that he was in fact suffering from a nosocomial infection caused by vancomycin-resistant Enterococcus (VRE).

Methods We performed a retrospective review of the patient's medical record.

Results Despite radiographic improvement, he did not improve clinically following immunosuppressive therapy including plasma exchange. Once meningitis was diagnosed and treated, he improved rapidly.

Conclusion The prospect of transverse myelitis may confound the diagnosis of VRE meningitis, a uniquely treated and potentially deadly infection.

\section{Background}

Transverse myelitis (TM) is an autoimmune inflammation of the spinal cord, often following a bacterial or viral infection. This causes rapid demyelination of the spinal cord leading to pain, paresthesias, muscle weakness, and/or paralysis below the level of inflammation.

TM may also develop in the setting of a systemic autoimmune disorder such as systemic lupus erythematosus (SLE). First-line therapies include intravenous glucocorticoids and immunomodulatory drugs such as cyclophosphamide. However, in refractory or rapidly decompensating patients, therapeutic plasma exchange (TPE) may be considered. ${ }^{1}$ Transverse myelitis is a Category II indication for TPE, meaning that it is appropriate for use as a second-line therapy. Level 1B evidence supports its use in this role. ${ }^{2}$

received

February 5, 2017

accepted

July 14, 2017
DOI https://doi.org/

10.1055/s-0037-1607280. ISSN 2193-6358.
Vancomycin-resistant Enterococcus (VRE) is a rare cause of meningitis, which is typically seen in patients with a history of recent neurosurgery. ${ }^{3}$ The etiology should be identified as soon as possible due to its lethality and relatively unique antimicrobial therapy of linezolid.

\section{Methods}

We performed a retrospective case review of the patient's record.

\section{Case Report}

The patient was a 32-year-old male who had been diagnosed with SLE in 2007 based on antinuclear antibody (ANA),

\footnotetext{
(C) 2017 Georg Thieme Verlag KG Stuttgart · New York
}

License terms

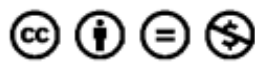


double-stranded DNA (dsDNA) antibody, low complement levels, and arthritis. Appropriate follow-up had been maintained until June 2012. Citing an inability to afford his medications, he remained untreated for at least a month prior to admission.

He presented to an outside hospital ER with a 2-day history of persistent headaches and dizziness. Physical exam revealed systolic blood pressure in excess of $200 \mathrm{~mm} \mathrm{Hg}$. CT scan showed hydrocephalus with fourth ventricular hemorrhage. MRI was performed to detect herniation and revealed a pons, which was T3 hyperintense. The differential diagnosis included acute inflammation, venous congestion, and central cord infarct.

A decompressive craniectomy was performed, and an external ventricular drain (EVD) was placed. A ventriculoperitoneal (VP) shunt was later placed. CT showed a stable fourth ventricle hemorrhage and a smaller ventricular system. He remained in an altered mental state and was unable to move his lower extremities.

Concurrently, his renal function declined. His creatinine initially improved from $2.56 \mathrm{mg} / \mathrm{dL}$ on admission to $1.25 \mathrm{mg} / \mathrm{dL}$ around the time of craniectomy, but he had been receiving dexamethasone perioperatively. His creatinine continued to increase to a peak of $3.85 \mathrm{mg} / \mathrm{dL}$. A renal biopsy demonstrated class IV lupus nephritis. Consequently, he was started on hemodialysis every 1 to 2 days that lasted for about 4 weeks.

Due to his lower extremity weakness and radiographical findings, a working diagnosis of transverse myelitis was made. The treatment regimen included cyclophosphamide, hydroxychloroquine, and therapeutic plasma exchange (TPE). TPE was performed daily for 5 days. Fresh frozen plasma was used as the replacement fluid due to his risk of intracranial hemorrhage.

MRI after his first TPE revealed decreased hyperintensity and swelling. His level of consciousness remained low; so, the search continued for other etiologies. Lumbar puncture revealed grampositive cocci; 2,156 neutrophils/ $\mu \mathrm{L}$; and $<1 \mathrm{mg} / \mathrm{dL}$ of glucose in his CSF. Once the diagnosis of bacterial meningitis (pending further characterization) was made, the immunosuppressive therapies were stopped, and he was started on vancomycin and ceftriaxone.

Enterococcus was cultured, and ceftriaxone was changed to ampicillin. Susceptibility testing led to a final identification of VRE, and he was switched to linezolid. Following the change in treatment, the patient showed improvement in consciousness and lower extremity function. He was discharged to inpatient rehabilitation and then to home.

\section{Discussion}

We report a case of SLE exacerbation complicated by nosocomial VRE meningitis. This infection muddled the clinical picture for what was presumed to be TM. This case shows that vigilance and astute clinical judgment are needed to care for a patient with a complex and evolving clinical course. Specifically, the continued brain-specific pathology made lumbar puncture a particularly important decision at the time, as it led to the identification of VRE. Due to the frequent insidious nature of VRE and its potential to be confused with other etiologies, maintaining and pursuing a broad differential diagnosis is essential to maximizing patient outcomes.

\section{References}

1 Krishnan C, Greenberg B, Kerr D. Transverse Myelitis. In: UpToDate, Gonzalez-Scarano F, ed. UpToDate, Waltham, MA. https://www. uptodate.com/contents/transverse-myelitis. Accessed on March 11, 2014

2 SchwartzJ, Winters JL, Padmanabhan A, et al. Guidelines on the use of therapeutic apheresis in clinical practice-evidence-based approach from the Writing Committee of the American Society for Apheresis: the sixth special issue. J Clin Apher 2013;28(03):145-284

3 Le J, Bookstaver PB, Rudisill CN, et al. Treatment of meningitis caused by vancomycin-resistant Enterococcus faecium: high-dose and combination daptomycin therapy. Ann Pharmacother 2010; 44(12):2001-2006 